\title{
Biopsy-induced inflammatory conditions improve endometrial receptivity: the mechanism of action
}

\author{
Y Gnainsky ${ }^{*}$, I Granot $^{1, *}$, P Aldo ${ }^{2}$, A Barash ${ }^{1}$, Y Or ${ }^{1}$, G Mor $^{2}$ and N Dekel \\ Department of Biological Regulation, The Weizmann Institute of Science, Rehovot, Israel, ${ }^{1}$ IVF Unit, \\ Department of Obstetric, and Gynecology, Kaplan Medical Center, Rehovot, Israel and ${ }^{2}$ Department of \\ Obstetric, and Gynecology, Yale University School of Medicine, New Haven, Connecticut, USA
}

Correspondence should be addressed to Y Gnainsky; Email: yulia.gnainsky@weizmann.ac.il

*(Y Gnainsky and I Granot contributed equally to this work)

\begin{abstract}
A decade ago, we first reported that endometrial biopsy significantly improves the success of pregnancy in IVF patients with recurrent implantation failure, an observation that was later confirmed by others. Recently, we have demonstrated that this treatment elevated the levels of endometrial pro-inflammatory cytokines and increased the abundance of macrophages (Mac) and dendritic cells (DCs). We therefore hypothesised that the biopsy-related successful pregnancy is secondary to an inflammatory response, and aimed at deciphering its mechanism of action. Supporting our hypothesis, we found that the pro-inflammatory TNF $\alpha$ stimulated primary endometrial stromal cells to express cytokines that attracted monocytes and induced their differentiation into DCs. These monocytederived DCs stimulated endometrial epithelial cells to express the adhesive molecule SPP1 (osteopontin (OPN)) and its receptors ITGB3 and CD44, whereas MUC16, which interferes with adhesion, was downregulated. Other implantation-associated genes, such as CHST2, CCL4 (MIP1B) and GROA, were upregulated by monocyte-derived Mac. These findings suggest that uterine receptivity is mediated by the expression of molecules associated with inflammation. Such an inflammatory milieu is not generated in some IVF patients with recurrent implantation failure in the absence of local injury provoked by the biopsy treatment.

Reproduction (2015) 149 75-85
\end{abstract}

\section{Introduction}

Human embryo implantation is a three-stage process that includes apposition, adhesion and invasion. During apposition, chemokines and cytokines, produced by the endometrial cells, guide the blastocyst to the site of implantation and the embryonic L-selectin binds to its ligands on the luminal epithelium, enabling the initial contact of the blastocyst with the uterus. During adhesion, the interaction between the blastocyst and the uterus is further established by binding of the epithelial adhesion molecules, such as intergrins, to their ligands on the trophoblast cells. Invasion of the trophoblast is regulated by its crosstalk with the different endometrial cell components (Lessey 2002, van Mourik et al. 2009).

Successful implantation is dependent on both, the development of high-quality embryo and the acquisition of endometrial receptivity. Although fertilisation rate is relatively high and embryo culture conditions are constantly being improved, implantation remains a rate-limiting step for the success of IVF treatments. A major reason for implantation failure of the in vitro grown embryo is still an inadequate uterine function
(Edwards 1994, Simon et al. 1998, Fatemi \& PopovicTodorovic 2013). In humans, the uterus becomes receptive during the mid-secretory phase (days 19-23) of the menstrual cycle, known as the window of implantation (WOI). This period is characterised by morphological and transcriptional changes in the endometrium such as, cell proliferation and their differentiation; accumulation of specific immune cells, macrophages (Mac), dendritic cells (DCs) and natural killer (NK) cells and expression of different cytokines/ chemokines and growth factors as well as adhesion molecules. All these changes facilitate the embryoendometrium interaction enabling implantation (van Mourik et al. 2009, Granot et al. 2012).

It was a decade ago that we first demonstrated that endometrial biopsies taken during the spontaneous cycle preceding the IVF treatment substantially increase the rates of implantation, clinical pregnancies and live births (Barash et al. 2003). Such favourable effect of local endometrial injury was later confirmed by other IVF clinics worldwide (Raziel et al. 2007, Zhou et al. 2008, Karimzadeh et al. 2009, Almog et al. 2010, Narvekar et al. 2010, Tiboni et al. 2011, Nastri et al. 2013). 
Moreover, two meta-analyses of the results of the above-mentioned studies as well as a randomised clinical trial recently published strongly support the notion that local endometrial injury significantly improves clinical pregnancy and live birth rates in the subsequent IVF cycle (El-Toukhy et al. 2012, Potdar et al. 2012, Gibreel et al. 2013). Furthermore, it has been recently shown that biopsy treatment has a positive effect on spontaneous pregnancies as well (Brinsden et al. 2009).

Investigating the mechanism by which the biopsy treatment increases endometrial receptivity, we previously demonstrated that the local injury induces an inflammatory response that is characterised by elevated levels of pro-inflammatory cytokines/chemokines, such as macrophage inflammatory protein (MIP)1B, tumour necrosis factor alpha (TNF $\alpha)$, growth-regulated oncogene alpha $(\mathrm{GRO} \alpha)$, SPP1 osteopontin (OPN) and IL15 (Gnainsky et al. 2010). In this study, we also demonstrated an increased abundance of specific $\mathrm{CD} 11 \mathrm{c}^{+} \mathrm{HLA}^{-D R^{+}}$ immune cells, representing Mac and DCs. Most importantly, we showed a positive correlation between the biopsy-induced pro-inflammatory cytokines and successful pregnancy, suggesting that these molecules may serve as possible biomarkers for implantation competence (Gnainsky et al. 2010). Taking the above-mentioned findings into consideration, we hypothesise that endometrial biopsy triggers an inflammatory response that induces the transition of the endometrium from its nonreceptive to its receptive state (Dekel et al. 2014).

The present study was designed to test this hypothesis. Due to the obvious ethical and technical limitations of investigating implantation in human in vivo, we employed an in vitro experimental model using primary human endometrial cells isolated from the biopsies taken from IVF patients as well as an endometrial cell line. We demonstrate that injury-induced pro-inflammatory cytokines secreted by the endometrial stromal cells (ESCs) attract monocytes and induce their differentiation into DC-like cells. Furthermore, monocyte-derived DCs directly affect ESCs and endometrial epithelial cells (EECs) to express a specific repertoire of chemokines and adhesion molecules that are involved in the uterineblastocyst interaction. We suggest that mechanical intervention by endometrial biopsy may be necessary in IVF patients with recurrent implantation failures for provoking the inflammatory response required for acquisition of endometrial receptivity.

\section{Materials and Methods}

\section{Patients and endometrial sample collection}

IVF patients, 22-39 years of age with menstrual cycles of 28-30 days and are good responders to the hormonal stimulation were selected. Patients with endometriosis and/ or hydrosalpinx were excluded. Endometrial samples of the functional layer of the endometrium were retrieved using a biopsy catheter (Pipelle de Cornier, Prodimed, Neuilly-enThelle, France) (Barash et al. 2003, Gnainsky et al. 2010). In each biopsy, the endometrial sample was taken from several spots. Biopsies were taken during the spontaneous menstrual cycle preceding the IVF cycle, on day 12, the non-receptive, and/or day 21 (WOI), the receptive phase of the cycle. The samples were immediately transferred into culture medium for the isolation of endometrial cells.

The protocol of this study has been approved by the Kaplan Medical Center Committee on the use of Human Subjects in Medical Research in accordance with the Helsinki declaration. Informed consent was received from all participants.

\section{Isolation of endometrial stromal and epithelial cells}

Endometrial cells were isolated as described previously (Gnainsky et al. 2010). In brief, fresh endometrial samples were minced into $1-2 \mathrm{~mm}$ fragments and subjected to enzymatic digestion using $2.5 \mathrm{mg} / \mathrm{ml}$ collagenase (Sigma-Aldrich, St. Louis, MO, USA) and DNase (Roche Applied Science, Penzberg, Germany) for $1.5 \mathrm{~h}$. The cells were then transferred through a $100 \mu \mathrm{m}$ cell strainer. EECs and ESCs were separated by their filtration though a $40 \mu \mathrm{m}$ cell strainer (BD Biosciences, San Jose, CA, USA) according to their size difference. The stromal cells were seeded and were used for further experiments on their first passage. Epithelial sheets were plated onto $30 \mathrm{~mm}$ plate and incubated for $30 \mathrm{~min}$ to allow the contaminating stromal cells to adhere. The media containing the non-adherent EECs were transferred to a 24-well plate for further experiments.

\section{Cell culture}

Freshly-isolated human ESCs and EECs were cultured in DMEM/F12 (Biological Industries, Kibbutz Beit Haemek, Israel). Human monocyte cell line (THP-1), blood-derived monocytes, Mac and DCs were cultured in complete RPMI-1640 (Biological Industries). All culture media were supplemented with $10 \%$ heat-inactivated foetal bovine serum (FBS, Gibco-Invitrogen, Carlsbad, CA, USA) and $100 \mathrm{U} / \mathrm{ml}$ penicillin/streptomycin (Biological Industries).

To induce an injury-like environment in vitro, ESCs were incubated with $25 \mathrm{nM} \mathrm{TNF} \alpha$ (Peprotech, Rocky Hill, NJ, USA), a pro-inflammatory cytokine that is known to initiate inflammatory response. To confirm its inflammatory effect, the conditioned medium (CM) was collected after $24 \mathrm{~h}$ of incubation and analysed for cytokine secretion profile using Multiplex analysis (see below).

\section{Migration assay}

The capacity of the TNF $\boldsymbol{\alpha}$-treated ESC to attract monocytes (monocyte cell line, THP-1) was tested using transwell migration assay. For this purpose, THP- 1 cells $\left(2 \times 10^{5}\right)$ were resuspended in RPMI medium and placed in transwell inserts with $5 \mu \mathrm{m}$ pore size (Millipore, Billerica, MA, USA). CM collected from either TNF $\boldsymbol{\alpha}$-treated or untreated ESC was placed in the bottom of the well. After $2 \mathrm{~h}$ of incubation, inserts were removed and the number of THP-1 cells migrated to the bottom of the well was assessed using haemacytometer. 


\section{Isolation of monocytes from peripheral blood}

Mononuclear cells were isolated from blood samples using FicollPaque PLUS (GE Healthcare, Buckinghamshire, UK), according to manufacturer's instructions. Leukocyte-enriched blood samples were purchased from the Blood Bank in Rabin Medical Center. The protocol of isolating cells from blood samples for research was approved by the Kaplan Medical Center Committee on the use of Human Subjects in Medical Research in accordance with the Helsinki declaration. The viability of the isolated mononuclear cells was assessed by staining with Trypan blue. The cells were then seeded in $10 \mathrm{~cm}$ cell culture plates. After $1 \mathrm{~h}$ of incubation, non-adhesive mononuclear cells were removed; attached monocytes were washed and further used for differentiation experiments. The purity of the isolated monocytes was tested by FACS as described below.

\section{Generation of Mac and DCs}

Human Mac and DCs were generated either from THP-1 monocyte cell line or from peripheral blood-derived monocytes using conventional in vitro differentiation methods as previously described (Berges et al. 2005, Hanlon et al. 2011). Briefly, to induce differentiation to DCs, monocytes were incubated with $100 \mathrm{ng} / \mathrm{ml}$ of IL4 and GM-CSF (Peprotech). To induce differentiation to Mac, monocytes were incubated with either $100 \mathrm{ng} / \mathrm{ml}$ M-CSF (Peprotech) or 20 nM TPA (Sigma-Aldrich). Following 3 and 6 days of incubation, cell phenotype was assessed by analysing the expression of specific surface molecules of Mac and DCs using FACS (see below). The CM was used for further experiments and was analysed for cytokine production using Multiplex Technology, as described below.

To study the effect of TNF $\alpha$-induced pro-inflammatory cytokines on monocyte differentiation, freshly isolated monocytes were incubated with CM from TNF $\alpha$-treated and untreated day 21-endometrial stromal cells. Following 3 days of culture, cell phenotype was assessed by FACS analysis. Monocyte-derived DCs and Mac served as reference for differentiation status.

\section{Multiplex analysis}

The levels of cytokines and chemokines secreted by the stromal and the immune cells to their culture medium were determined by multiplex analysis using the BioPlex assay (Bio-Rad, Hercules, CA, USA), in which Luminex 100 IS system (Luminex, Austin, TX, USA) was used for detection and analysis (Aldo et al. 2013).

\section{Analysis of expression of implantation-associated genes in endometrial cells}

Human ESCs and EECs isolated from day 12 endometrial samples representing non-receptive endometrium were treated with or without $\mathrm{CM}$ collected from different types of the immune cells (monocytes, Mac and DCs). After 24 h of culture, RNA was extracted using the PerfectPure RNA Cell kit (5 Prime, Gaithersburg, MD, USA). The expression of implantation associated genes was analysed by quantitative PCR. For the analysis of protein expression, epithelial cells were grown on round coverslips placed in 24-well plates with or without immune cell CM. After $48 \mathrm{~h}$ of incubation, the cells were fixed with $4 \%$ paraformaldehyde for $10 \mathrm{~min}$ and immunofluorescent staining was performed as described below.

\section{Quantitative real-time PCR}

Primers for quantitative real-time PCR (qPCR) were designed by Primer Express software (Applied Biosystems, Foster City, CA, USA) and analysed using the BLAT-UCSC Genome Browser for their specificity. Relative quantification of the mRNA was carried out using SYBR Green PCRMaster Mix (Applied Biosystems) in theStepOne system v2.1 (Applied Biosystems). As internal control, hypoxanthine phosphor-ribosyltransferase 1 (HPRT1) was amplified in parallel for each sample and used for normalisation. The results of $\mathrm{qPCR}$ are presented as a fold change (mean \pm s.E.M.) normalised to control samples.

\section{FACS analysis}

Immune cells were incubated with human immunoglobulin for $10 \mathrm{~min}$ and stained with fluorescent antibodies against specific markers of monocytes and monocyte-derived Mac and DCs, such as anti-CD14 (Milteney, Bergisch Gladbach, Germany), antiCD11c (Becton-Dickinson, San Jose, CA, USA), anti-HLA-DR and anti-CD1a (Biolegend, San Diego, CA, USA). After 30 min of incubation at $4{ }^{\circ} \mathrm{C}$, the cells were washed and analysed by FACSsort flow cytometer (Becton-Dickinson). Unlabelled cells and the respective isotype antibodies were used as control. The results were analysed using FCS express, version 3 software.

\section{Immunofluorescent staining}

For immunofluorescence staining, epithelial cells were fixed with 4\% PFA for 10 min, blocked with 10\% serum (Vector, Burlingame, CA, USA), and incubated overnight with mouse anti-human CD44 antibody (1:50; Novus Biologicals, Littleton, CO, USA) or mouse anti-human MUC16 (1:50; Abcam, Cambridge, MA, USA). The next day, the coverslips were washed and cy3-labelled

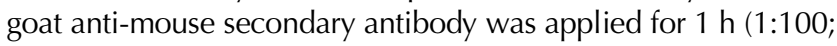
Jackson, West Grove, PA, USA). After washing, the nucleus were stained with Hoechst (1:2000; Sigma-Aldrich) for $1 \mathrm{~min}$ and then coverslips were mounted with Vectashield medium (Vector).

\section{Statistical analysis}

Statistical analysis was performed using computerised software GraphPad Prism (San Diego, CA, USA) and Statistica version 10 (Tulsa, OK, USA). The results have been analysed using nonparametric tests. Mann-Whitney $U$ test was used for comparison between two groups and Kruskal-Wallis test followed by Tukey's test for multiple comparisons. $P<0.05$ was considered statistically significant.

\section{Results}

\section{TNF $\alpha$-treated endometrial cells secret chemokines that attract monocytes}

We have previously demonstrated that in addition to its effect on cytokine production, endometrial injury 
induces a local increase in monocyte-derived immune cells, such as DCs and Mac (Gnainsky et al. 2010). We therefore hypothesised that the endometrial biopsy provokes an inflammatory response that stimulates the cells to secrete chemokines, which in turn recruit immune cells from the circulation to the site of injury. To test this hypothesis, we assessed the ability of endometrial cells to recruit monocytes in vitro. For this purpose, we isolated stromal cells from endometrial samples recovered from IVF patients on days 12 and 21
(A)
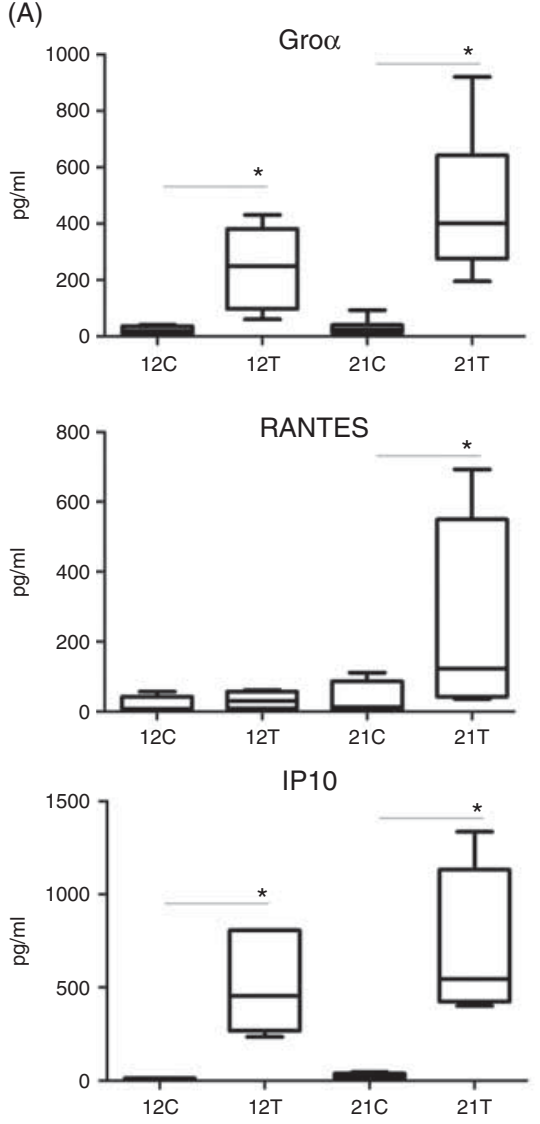

(B)

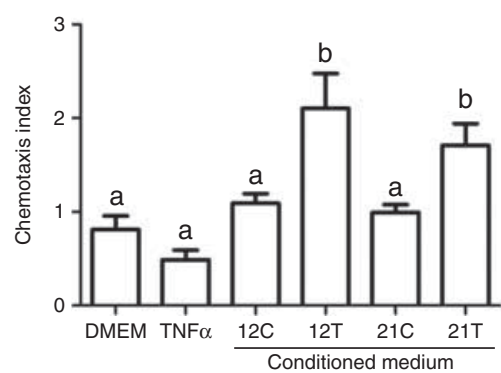

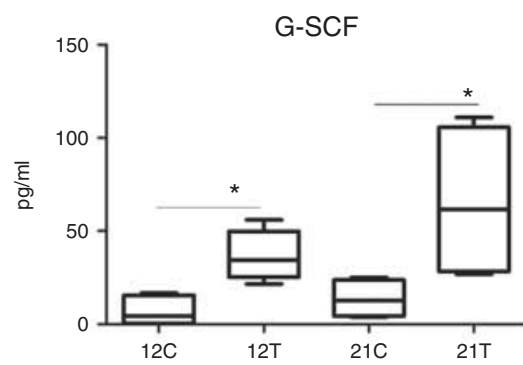
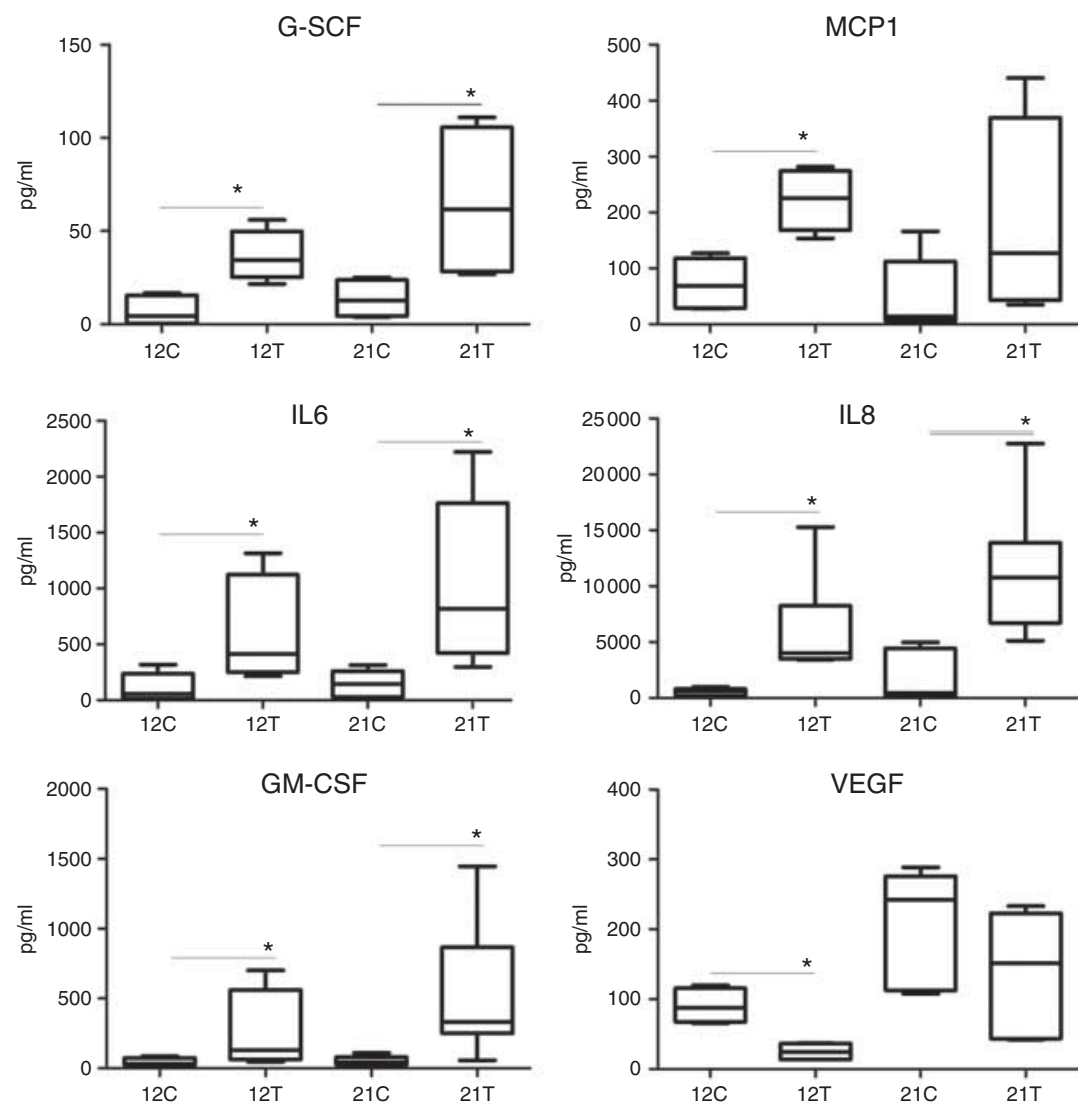

(C)

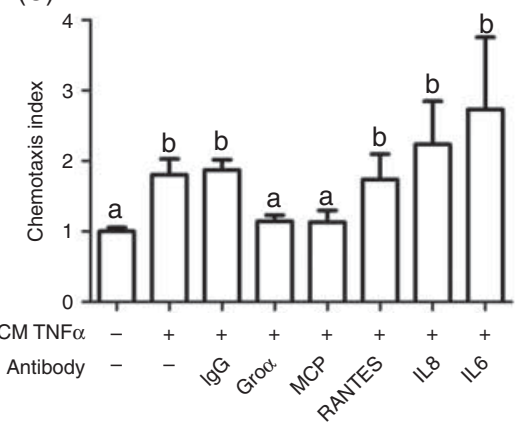

(D)

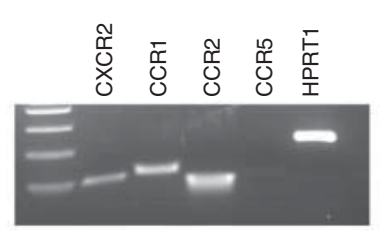

Figure $1 \mathrm{TNF} \alpha$ stimulates endometrial stromal cells to produce pro-inflammatory cytokines that attract monocytes. (A) Quantitative analysis of cytokine secretion by TNF $\alpha$-treated $(\mathrm{T})$ and untreated $(\mathrm{C})$ stromal cells isolated from endometrial samples recovered on days 12 and 21 of the menstrual cycle. The box plot horizontal lines represent the median and the 25 th to 75 th percentile ( $n=4-5$ independent experiments). ${ }^{*} P<0.05$, Kruskal-Wallis test, followed by Tukey's test. (B) Migration of THP-1 cells in response to $20 \mathrm{ng} / \mathrm{ml} \mathrm{TNF} \alpha$, conditioned medium of TNF $\alpha$-treated and untreated stromal cells isolated from day-12 (12T and 12C respectively) and conditioned medium of TNF $\alpha$-treated and untreated stromal cells isolated from 21 endometrial samples (21T and 21C respectively). Chemotaxis index represents the number of migrating cells under treatment with conditioned medium, divided by the number of migrating cells in the presence of cell-free medium (DMEM). Columns represent the average of three independent experiments \pm S.E.M. Small cap letters indicate significant differences based on Kruskal-Wallis test followed by Tukey's test $(P<0.05)$. (C) Migration of THP-1 cells in response to conditioned medium of TNF $\alpha$-treated stromal cells (CM TNF $\alpha$ ) following neutralization of selected cytokines. ( $n=5$ independent experiments). Small cap letters indicate significant differences based on Kruskal-Wallis test followed by Tukey's test $(P<0.05)$. (D) PCR analysis for the relevant chemokine receptors (CXCR2, CCR1, CCR2, and CCR5) expressed by THP-1 cells. Hypoxanthine phosphor-ribosyltransferase 1 (HPRT1) was used as a housekeeping gene. 
of their spontaneous menstrual cycle. Inflammation was induced by incubating these cells for $24 \mathrm{~h}$ with $25 \mathrm{ng} / \mathrm{ml}$ $\mathrm{TNF} \alpha$ (one of the cytokines that initiate an inflammatory response). CM was then collected and tested for cytokine profile and its ability to trigger monocyte migration.

Analysis of the cytokine profile of the CM collected from TNF $\alpha$-treated stromal cell revealed increased levels of RANTES, GRO $\alpha$, IL8, IL6, monocyte chemotactic protein 1 (MCP1), GM-CSF, interferon $\gamma$-induced protein (IP-10) and G-CSF, whereas the levels of VEGF were decreased by this treatment (Fig. 1A). This response of stromal cells to TNF $\alpha$ was similar in cells isolated from endometrial samples taken on both days 12 and 21 . Analysis of the cytokine RNA levels showed compatible results (data not shown).

The ability of CM collected from TNF $\alpha$-treated stromal cells to attract monocytes was tested by the transwell migration assay. We found a higher number of monocytes (THP-1 cells) recruited by CM from TNF $\alpha$ treated cells as compared with CM from untreated cells (Fig. 1B). To identify which of the chemokines in the TNF $\alpha$-treated stromal-cell CM attract monocytes, another set of migration assays was performed with the addition of relevant antibodies to neutralise specific cytokines. We found that anti-MCP1 and anti-GRO $\alpha$ antibodies inhibited the THP-1 cell migration (Fig. 1C), suggesting that these chemokines could be directly involved in monocyte recruitment in response to the endometrial biopsy-induced inflammation. Complimentary experiments demonstrated that THP- 1 cells do express the receptors for these cytokines (Fig. 1D).

\section{TNF $\alpha$-treated endometrial cells secrete chemokines that induce monocyte differentiation}

Monocyte differentiation into Mac and DCs is determined by the specific tissue microenvironment (Geissmann et al. 2010). We therefore assumed that differentiation of the recruited monocyte into uterine DCs or Mac is mediated by the cytokines secreted by the TNF $\alpha$-treated endometrial stromal cells. In our next experiments, we studied the effect of $\mathrm{CM}$ collected from TNF $\alpha$-stimulated stromal cells on monocyte differentiation. For this purpose, we evaluated the expression of the commonly used, Mac and DC surface markers (HLA-DR, CD14, CD11c, and CD1a) in response to TNF $\alpha$-treated and untreated (control) stromal cell CM. For reference, monocyte-derived DCs and Mac that underwent in vitro differentiation induced by GM-CSF and IL 4 or by M-CSF, respectively, were employed. FACS analysis revealed that monocytes incubated with TNF $\alpha$-treated stromal cell CM exhibited a DC-like cell phenotype (Fig. 2). Specifically, CD14 expression was reduced (Fig. 2A and C), CD1a expression was elevated (Fig. 2B and D), and HLA-DR expression was slightly decreased (Fig. 2E) with no change observed in CD11c
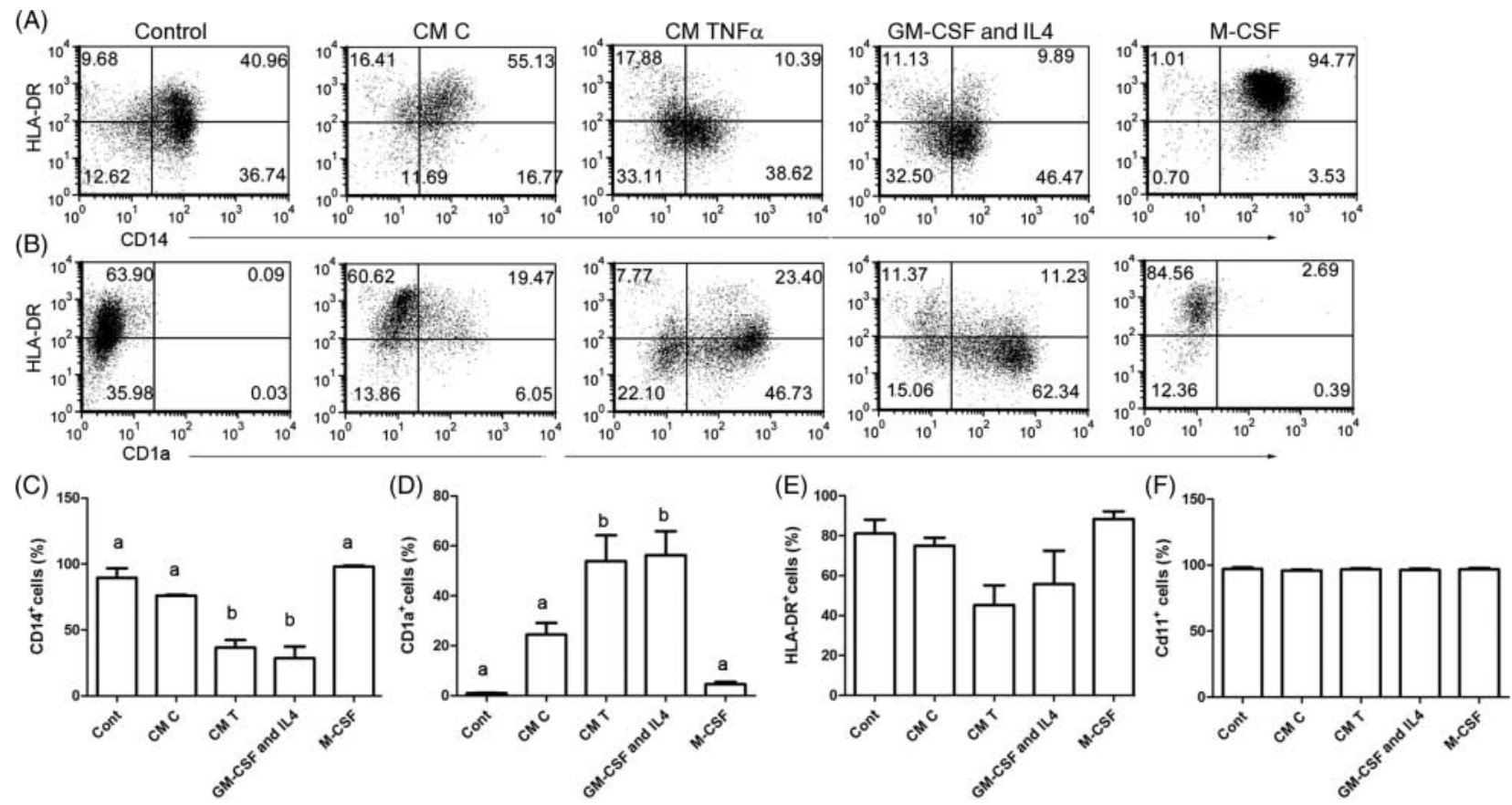

Figure 2 Conditioned medium (CM) collected from TNF $\alpha$-treated stromal cells induces differentiation of blood-derived monocytes into DC-like cells. (A and B) A representative FACS analysis of monocyte differentiation using the specific cell surface markers for Mac and DCs: HLA-DR, CD14, CD1a, and Cd11c. Freshly isolated monocytes (control) were cultured for 3 days with CM from TNF $\alpha$-treated (CM T) and untreated (CM C) stromal cells. As a positive control, cells were incubated with GM-CSF and IL4 for DC differentiation and with M-CSF for Mac differentiation. (C, D, E and F) Quantification of the cell surface marker expression determined by FACS as shown in A and B. The results represent three independent experiments each of which conducted using CM from endometrial stromal cells of a different patient. Small cap letters indicate significant differences based on Kruskal-Wallis test followed by Tukey's test $(P<0.05)$. 

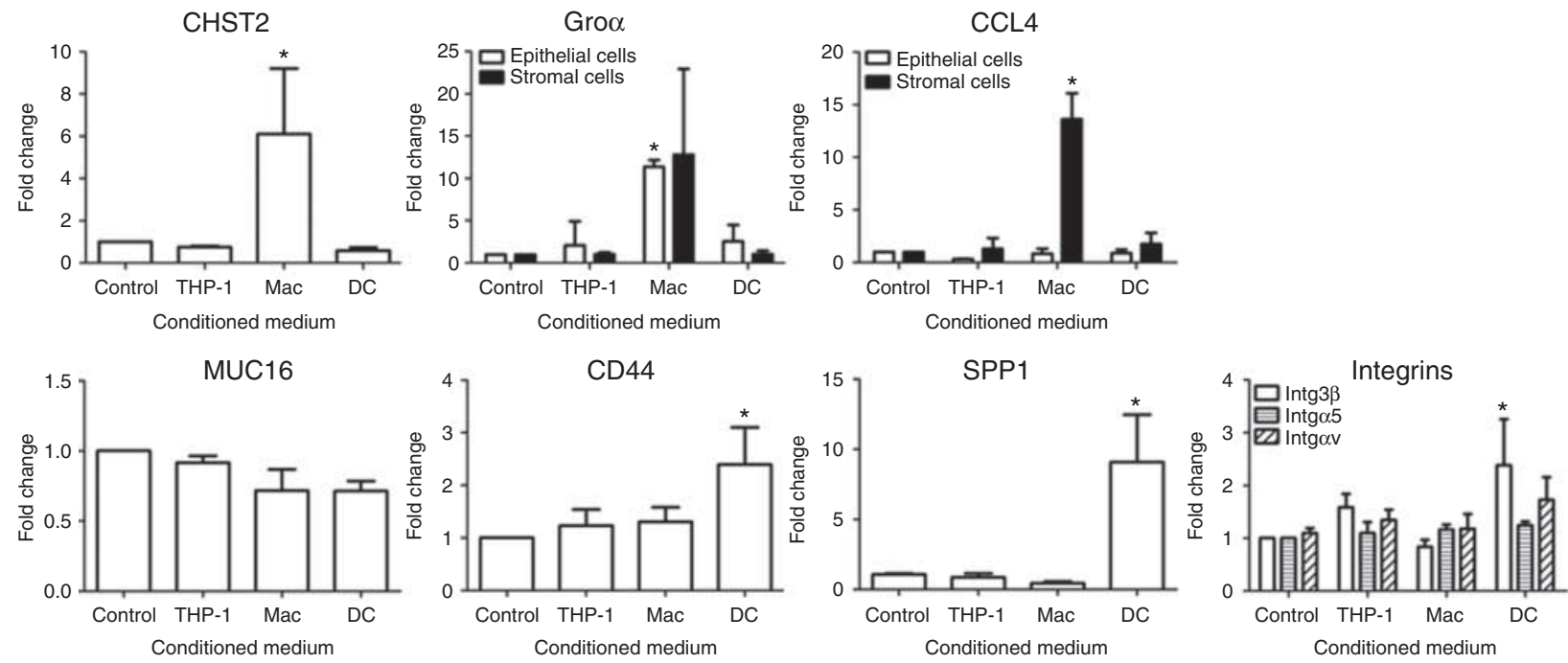

Figure 3 Regulation of the expression of implantation-associated genes in endometrial stromal and epithelial cells by THP-1 cell-derived Mac and DCs. Freshly isolated, either stromal or epithelial cells, from day-12 endometrial samples were treated with either RPMI-culture medium (control) or with conditioned medium recovered from either monocytes (THP-1), Mac or DCs. The effect of these immune cells on the expression of the implantation-associated genes was tested by qPCR. CCL4 and GRO $\alpha$ were analysed in both epithelial and stromal cells (white and black columns respectively), while CHST2, SPP1, integrins $\alpha$ vand $\beta 3$ and MUC16 were analysed only in the epithelial cells. The results of qPCR are normalized to hypoxanthine phosphor-ribosyltransferase 1 and represent the mean \pm S.E.M. of five independent experiments, each of which was conducted on cells of a different patient. ${ }^{*} P<0.05$, Kruskal-Wallis test.

expression (Fig. 2F). The phenotype of monocytes that were incubated with $\mathrm{CM}$ of untreated cell was characterised by HLA-DR ${ }^{\text {high }} \mathrm{CD} 11 \mathrm{c}^{+} \mathrm{CD} 14^{+} \mathrm{CD} 1 \mathrm{a}^{-}$(Fig. 2), a phenotype that is associated with tissue Mac.

To further characterise this effect and remove any potential contaminant immune cell present in the primary cultures, we carried out experiments as described above using the well-characterised endometrial stromal cell line HESC (Krikun et al. 2004). The CM collected from TNF $\alpha$-treated HESC induced differentiation that was similar to that described above for the primary cultures (data not shown), indicating that the TNF $\alpha$-treated stromal cells are directly involved in monocyte differentiation into DCs.

\section{Monocyte-derived DCs directly affect endometrial cell receptivity}

We assumed that the recruited immune cells are involved in the acquisition of endometrial receptivity. We therefore studied their direct effect on endometrial cells in vitro. For this purpose, freshly isolated endometrial stromal and epithelial cells were cultured with CM collected from either Mac or DCs derived from either THP-1 or peripheral blood monocytes. Specifically, we tested the effect of the immune cell $\mathrm{CM}$ on endometrial cell expression of implantation-associated genes. We found that CM collected from both THP-derived DCs and Mac affect the expression of genes that are involved in the embryo-endometrium interaction and that this effect is cell and gene specific (Fig. 3). Mac CM significantly increased the expression of CHST2 that is responsible for the production of the functional L-selectin ligands in the epithelial cells. This $\mathrm{CM}$ also elevated CCL4 (MIP1B) and GROA mRNA in the stromal cells. CM collected from DCs regulated the expression of genes that are involved in the adhesion of the blastocyst to the luminal uterine surface. Specifically, mRNA expression of SPP1 and, its receptors, ITGB3 and $C D 44$, were upregulated, whereas the expression of the adhesion inhibitory molecule MUC16 was downregulated. A similar effect on the adhesion molecule expression was exerted by $\mathrm{CM}$ collected from the blood monocyte-derived DCs (Fig. 4A). Complementary immunofluorescent staining confirmed these findings at the protein level (Fig. 4B).

Multiplex analysis of the cytokine profile of DC-CM detected a variety of cytokines such as RANTES, CCL4, GRO $\alpha$, IL6, MCP1, GM-CSF, IP10, and SPP1 that may be potential mediators of the effect of DCs described above.

\section{Discussion}

This study unravels the role of pro-inflammatory cytokines and immune cells in preparing the endometrium for implantation. Specifically, we demonstrate herein that inflammatory conditions achieved by exposure to TNF $\alpha$ stimulate the endometrial stromal cells to produce a set of cytokines capable of stimulating monocyte migration and their differentiation into DCs. These immune cells in turn modify the expression of specific adhesion molecules by the endometrial epithelium.

Accumulating evidence that endometrial injury improves the rate of implantation generated by us and 

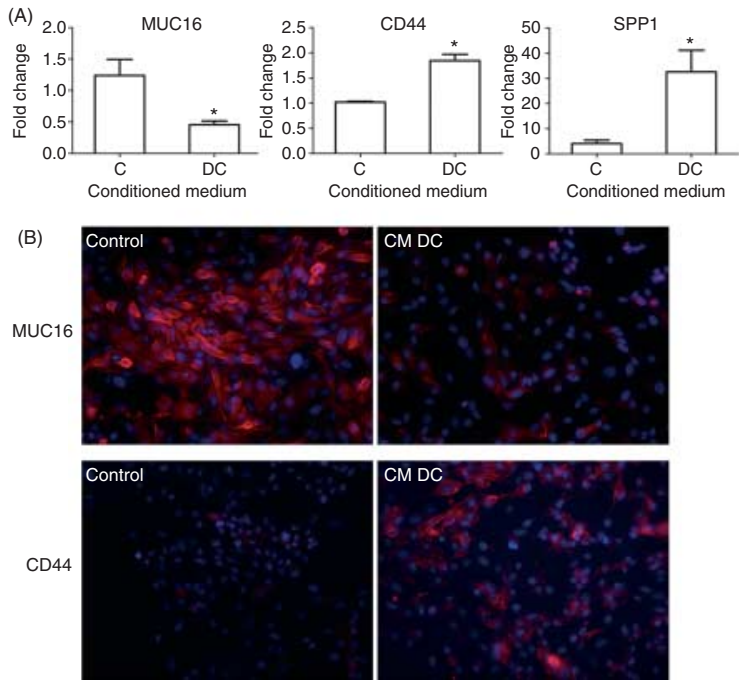

Figure 4 The expression of adhesion molecules by endometrial epithelial cells is regulated by blood monocyte-derived DCs. Freshly isolated day-12 epithelial cells were treated with either control culture medium (C) or with conditioned medium collected from cultures of blood monocyte-derived DCs (DC). (A) Expression of mucin 16 (MUC16), osteopontin (SPP1) and CD44 was analysed by qPCR. The results of qPCR are normalized to hypoxanthine phosphor ribosyltransferase 1 and represent the mean \pm s.E.M. of five independent experiments, each of which was conducted on cells of a different patient. ${ }^{*} P<0.05$, Mann-Whitney $U$ test. (B) Complementary analysis of DC modulation of MUC16 and CD44 was performed by immunofluorescence. Blue staining (Hoechst) represents cell nuclei; red staining represents either CD44 or MUC16. CM DC-dendritic cell conditioned medium.

others endorsed a role of inflammation in acquisition of uterine receptivity (Barash et al. 2003, Raziel et al. 2007, Zhou et al. 2008, Karimzadeh et al. 2009, Dekel et al. 2010, Narvekar et al. 2010, Tiboni et al. 2011, Granot et al. 2012, Nastri et al. 2013). Furthermore, the strong correlation between successful implantation and increased levels of endometrial pro-inflammatory cytokines, as well as elevated abundance of immune cells, following endometrial local injury (Gnainsky et al. 2010) reinforces this notion. The results of this study indicate that the pro-inflammatory $\mathrm{TNF} \alpha$, which is upregulated upon endometrial biopsy (Gnainsky et al. 2010), could contribute to the injury-induced rise in endometrial proinflammatory cytokines. Previous studies demonstrated that some of these cytokines are directly involved in implantation. For instance, IL6 and CCL4 were found as the effective chemoattractants for trophoblast cells in human and IP10 has been shown to take part in the regulation of blastocyst migration, apposition and initial adhesion (Hannan et al. 2006, Dominguez et al. 2008, Sela et al. 2013). High levels of CCL4 and IP10 were positively associated with elevated rates of implantation in IVF patients (Boomsma et al. 2009, Gnainsky et al. 2010). Another cytokine, GM-CSF, was shown to be necessary for the growth and survival of the foetus
(Seymour et al. 1997). Its addition to human embryo culture medium significantly increased pregnancy and live birth rates (Ziebe et al. 2013). The production of the injury-induced cytokines IP10, MCP1, and CCL4 was shown to be regulated by estrogen (Boucher et al. 2000, Kitaya et al. 2003, Sentman et al. 2004, Carlino et al. 2008). Local injury of the endometrium during the proliferative phase of the menstrual cycle substantially increases endometrial expression of estrogen receptors in the following secretory phase ( $\mathrm{Li} \&$ Hao 2009). Therefore, this reciprocal regulation of estrogen and pro-inflammatory cytokines may indicate that the estrogen-induced proliferation in the endometrium is mediated by cytokines. Taken together, these findings could possibly suggest that in IVF patients with recurrent implantation failures, estrogen is insufficient for provoking endometrial receptivity. In these patients, mechanical intervention by endometrial biopsy is necessary to elicit the inflammatory response required for successful implantation.

We show herein that under a TNF $\alpha$-provoked inflammatory state, endometrial stromal cells secrete cytokines that stimulate monocyte migration specifically identifying MCP1 and GRO $\alpha$ as the cytokines that elicit this response. These findings are in line with our previous demonstrations that the endometrial biopsy-induced increase in pro-inflammatory cytokines is associated with accumulation of $\mathrm{HLA}_{-} \mathrm{DR}{ }^{+} \mathrm{CD} 11 \mathrm{c}^{+} \mathrm{CD}^{-} 4^{-}$(DCs) and $\mathrm{HLA}_{-\mathrm{DR}}^{+} \mathrm{CD} 11 \mathrm{c}^{+} \mathrm{CD} 14^{+}$(Mac) cells in the endometrium (Gnainsky et al. 2010). They also agree with previous findings that pro-inflammatory cytokines act as chemokines that induce the recruitment of monocytes to injured tissues (Schober \& Weber 2005, Shi \& Pamer 2011). We further found that the CM recovered from TNF $\alpha$-treated endometrial stromal cells provides proinflammatory environment that stimulates the differentiation of monocytes into DC-like cells. Differentiation of the recruited monocytes into specific immune cells, such as Mac and DCs, that is dependent on environmental signals has been described also in previous reports (Chapuis et al. 1997, Iwamoto et al. 2007).

It has been reported that the abundance of DCs and Mac in human endometrium reaches its highest levels during the WOI (Rieger et al. 2004, Kämmerer 2005, Laskarin et al. 2007). These reports go in line with our previous demonstration that an increased abundance of these cells in the endometrium following biopsy treatment significantly associated with improved pregnancy rates (Gnainsky et al. 2010). In support of these reports, studies carried out in mice provided strong evidence for the indispensability of DCs in implantation (Blois et al. 2004, Plaks et al. 2008). Accordingly, it has been shown that therapy by DCs administration significantly decreased the rate of spontaneous resorption of mouse embryos (Blois et al. 2004). These studies further demonstrate that DCs and Mac secrete an array of cytokines/chemokines and enzymes that may play a 
role in tissue remodeling and angiogenesis, regulating trophoblast invasion and decidualisation (Goetzl et al. 1996, Plaks et al. 2008, David Dong et al. 2009, Blois et al. 2011). However, the specific role of DCs and Mac in preparation of the human uterus for implantation remained unclear. Our attempts to decipher the mechanism by which monocyte-derived cells affect the capacity of endometrial epithelium to interact with the embryo revealed that $\mathrm{CM}$ collected from Mac elevated mRNA levels of CHST2 and CCL4 in epithelial and stromal endometrial cells respectively. These genes were proposed to be associated with the initial contact of the blastocyst with the endometrial epithelium. It was shown that CHST2 is responsible for the production of the functional endometrial L-selectin ligands that interact with embryonic L-selectins (Genbacev et al. 2003). In human endometrium, elevated level of L-selectin ligands has been associated with improved implantation (Margarit et al. 2009). In this context, decreased expression of CHST2 was associated with infertility (Margarit et al. 2009). Another molecule apparently involved in embryouterine interaction is CCL4. Among its other functions, this cytokine has the ability to attract blastocyst to the site of implantation (Hannan et al. 2006). We previously found a strong correlation between CCL4 levels in the endometrium and successful pregnancy in biopsy-treated IVF patients, suggesting that CCL4 could potentially serve as a biomarker for the prediction of implantation competence (Gnainsky et al. 2010).

Other molecules that could be possibly involved in the attachment of the embryo to the uterine wall and are directly modified by monocyte-derived cells are the antiadhesive MUC16 and the adhesion molecules SPP1 and its receptors, ITG $\beta 3$, and $C D 44$. The membrane glycoprotein MUC16 is expressed in the non-receptive area of the luminal uterine surface, preventing undesirable embryouterine interaction and its misplaced implantation (Gipson et al. 2008). Removal of MUC16 from epithelial cells in vitro increased the adhesion of trophoblast cells (Gipson et al. 2008). Our results suggest that DCs positively affect the adhesive capacity of EECs by downregulating the MUC16 along with upregulation of the SPP1, ITG $\beta 3$ and CD44. These findings agree with the previous reports of the association between an increase of
(A)

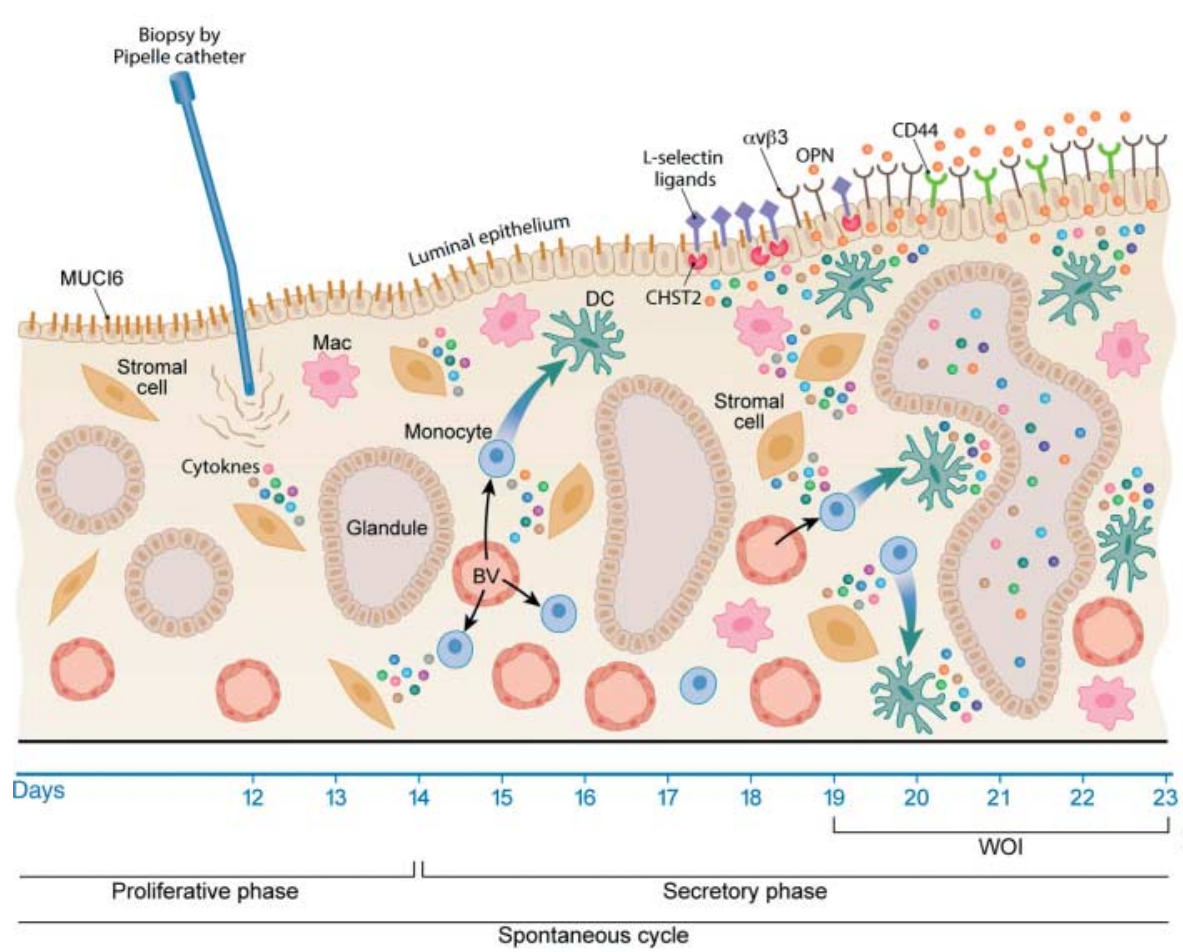

(B)

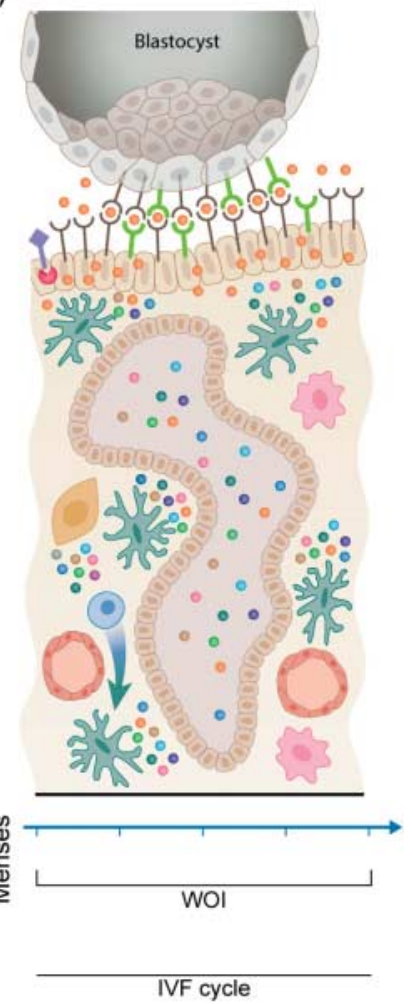

Figure 5 The postulated model of the injury-induced events in the endometrium that lead to an increase in uterine receptivity. (A) Endometrial biopsy, performed during the proliferative phase of the spontaneous menstrual cycle, triggers the secretion of a repertoire of pro-inflammatory cytokines by the stromal cells. These cytokines recruit monocytes from the circulation and further induce their differentiation into DCs and Mac. These immune cells, in turn, secrete a set of cytokines that trigger the stromal and epithelial cells to express specific implantation-associated genes. Specifically, Mac induces the epithelial cells to express CHST2 and the subsequent production of functional L-selectin ligands whereas DCs upregulate the epithelial cell production of the adhesion molecule SPP1 and its receptors ITG $\alpha$ v $\beta 3$ and CD44 with a concomitant downregulation of the anti-adhesive molecule MUC16. (B) The recruited monocytes reside in the endometrial tissue and elicit the effects mentioned above on endometrial receptivity facilitating implantation at the following IVF cycle. 
these adhesive molecules and the endometrial receptive phase (Lessey et al. 1992, Apparao et al. 2001, Afify et al. 2006, Horcajadas et al. 2007). Since in addition to the EECs, SPP1 receptors are expressed on the trophoblast cells, it was suggested that SPP1 serves as a bridge that links the luminal uterine cells with the blastocyst (Apparao et al. 2001, Johnson et al. 2003), thus playing a crucial role in establishing the embryo-endometrium interaction. Studies carried out in mice and rabbits indeed showed that using specific antibodies for functional blocking of endometrial, either ITG $\alpha$ v $\beta 3$ or SPP1 in vivo significantly reduced the number of implantation sites (Illera etal. 2000, 2003, Liu et al. 2013). Further in vitro experiments demonstrated that blastocysts failed to attach to EECs pre-treated with ITG $\beta 3$ siRNA (Kaneko et al. 2011).

It is important to note that the favourable effect of the biopsy treatment on endometrial receptivity was observed in the IVF treatment performed in the following cycle (Barash et al. 2003). This delayed response is dependent on 'tissue memory' apparently contributed by the recruited monocytes that reside in the tissue for several months, during which time they can constitute distinct-resident tissue Mac and DC populations (Chomarat et al. 2003, Luster et al. 2005, McIntire et al. 2008). In this context, it should be noted that during menstruation the endometrial thickness is reduced due to the loss of fluid and shrinkage of the spongy layer, whereas most of the stroma and apparently the embedded immune cells remain intact (Brenner \& Slayden 1994). Under these conditions, the modified properties of endometrial stromal and epithelial cells, provoked by the biopsy treatment could be preserved for the following IVF cycle.

In summary, our findings support the idea that the injury-induced improved endometrial receptivity in IVF patients with repeated implantation failure is mediated by an inflammatory response that comprises the cooperation between the two endometrial compartments, stromal and epithelial cells, orchestrated by the recruited immune cells Mac and DCs. The following is our proposed model for the series of events that occur in response to the endometrial injury (Fig. 5). Endometrial biopsy upregulates the expression of pro-inflammatory cytokines that recruit monocytes/Mac to the site of injury further inducing their differentiation into Mac and DCs that in turn trigger the stromal and epithelial cells to express some particular implantation-associated genes, which are involved in the apposition and adhesion of the blastocyst. Specifically, Mac-induced CHST2 together with CCL4, produced by the stromal cells, guide the blastocyst to the desired implantation site, whereas DCs-induced upregulation of epithelial cell adhesion molecules SPP1, ITG $\alpha v \beta 3$, and CD44 in combination with downregulation of the anti-adhesive MUC16 enable the attachment of the blastocyst to the uterine epithelial surface, facilitating implantation. These events probably do not take place in patients with repeated implantation failures in the absence of the endometrial biopsy treatment.

\section{Declaration of interest}

The authors declare that there is no conflict of interest that could be perceived as prejudicing the impartiality of the research reported.

\section{Funding}

This study was funded by Dwek Fund for Biomedical Research, United States - Israel Binational Science foundation and by the Chief Scientist, Israel Ministry of Health.

\section{Author contribution statement}

Y Gnainsky designed and conducted the experiments, analysed and interpreted the data, wrote and revised the manuscript. I Granot collected the samples, designed the experiments, analysed and interpreted the data, wrote and revised the manuscript. P Aldo conducted the experiments, analysed the data and revised the article. A Barash and $Y$ Or recruited patients for the study, performed clinical procedures and sample collection and revised the manuscript. N Dekel and G Mor interpreted the data, contributed to discussion, and revised the manuscript. All authors gave their final approval of the version to be published.

\section{References}

Afify AM, Craig S \& Paulino AF 2006 Temporal variation in the distribution of hyaluronic acid, CD44s, and CD44v6 in the human endometrium across the menstrual cycle. Applied Immunohistochemistry \& Molecular Morphology 14 328-333. (doi:10.1097/00129039-200609000-00012)

Aldo PB, Craveiro V, Guller S \& Mor G 2013 Effect of culture conditions on the phenotype of THP-1 monocyte cell line. American Journal of Reproductive Immunology 70 80-86. (doi:10.1111/aji.12129)

Almog B, Shalom-Paz E, Dufort D \& Tulandi T 2010 Promoting implantation by local injury to the endometrium. Fertility and Sterility 94 2026-2029. (doi:10.1016/j.fertnstert.2009.12.075)

Apparao KB, Murray MJ, Fritz MA, Meyer WR, Chambers AF, Truong PR \& Lessey BA 2001 Osteopontin and its receptor $\alpha v \beta(3)$ integrin are coexpressed in the human endometrium during the menstrual cycle but regulated differentially. Journal of Clinical Endocrinology and Metabolism 86 4991-5000.

Barash A, Dekel N, Fieldust S, Segal I, Schechtman E \& Granot I 2003 Local injury of the endometrium doubles the incidence of successful pregnancies in patients undergoing in-vitro fertilization. Fertility and Sterility 79 1317-1322. (doi:10.1016/S0015-0282(03)00345-5)

Berges C, Naujokat C, Tinapp S, Wieczorek H, Hoh A, Sadeghi M, Opelz G \& Daniel V 2005 A cell line model for the differentiation of human dendritic cells. Biochemical and Biophysical Research Communications 333 896-907. (doi:10.1016/j.bbrc.2005.05.171)

Blois SM, Alba Soto CD, Tometten M, Klapp BF, Margni RA \& Arck PC 2004 Lineage, maturity, and phenotype of uterine murine dendritic cells throughout gestation indicate a protective role in maintaining pregnancy. Biology of Reproduction 70 1018-1023. (doi:10.1095/biolreprod.103. 022640)

Blois SM, Klapp BF \& Barrientos G 2011 Decidualization and angiogenesis in early pregnancy: unravelling the functions of DC and NK cells. Journal of Reproductive Immunology 88 86-92. (doi:10.1016/j.jri.2010. $11.002)$ 
Boomsma CM, Kavelaars A, Eijkemans MJ, Lentjes EG, Fauser BC, Heijnen CJ \& Macklon NS 2009 Endometrial secretion analysis identifies a cytokine profile predictive of pregnancy in IVF. Human Reproduction 24 1427-1435. (doi:10.1093/humrep/dep011)

Boucher A, Mourad W, Mailloux J, Lemay A \& Akoum A 2000 Ovarian hormones modulate monocyte chemotactic protein-1 expression in endometrial cells of women with endometriosis. Molecular Human Reproduction 6 618-626. (doi:10.1093/molehr/6.7.618)

Brenner RM \& Slayden OV 1994 Cyclic changes in the primate oviduct and endometrium. In Knobil E, Neill, J.D. (ed) The Physiology of Reproduction, Raven Press, Ltd, New York.

Brinsden PR, Alam V, de Moustier B \& Engrand P 2009 Recombinant human leukemia inhibitory factor does not improve implantation and pregnancy outcomes after assisted reproductive techniques in women with recurrent unexplained implantation failure. Fertility and Sterility 91 1445-1447. (doi:10.1016/j.fertnstert.2008.06.047)

Carlino C, Stabile H, Morrone S, Bulla R, Soriani A, Agostinis C, Bossi F, Mocci C, Sarazani F, Tedesco F et al. 2008 Recruitment of circulating NK cells through decidual tissues: a possible mechanism controlling NK cell accumulation in the uterus during early pregnancy. Blood 111 3108-3115. (doi:10.1182/blood-2007-08-105965)

Chapuis $F$, Rosenzwajg $M$, Yagello $M$, Ekman $M$, Biberfeld $P$ \& Gluckman JC 1997 Differentiation of human dendritic cells from monocytes in vitro. European Journal of Immunology 27 431-441. (doi:10.1002/eji.1830270213)

Chomarat P, Dantin C, Bennett L, Banchereau J \& Palucka AK 2003 TNF skews monocyte differentiation from macrophages to dendritic cells. Journal of Immunology 171 2262-2269. (doi:10.4049/jimmunol.171.5. 2262)

David Dong ZM, Aplin AC \& Nicosia RF 2009 Regulation of angiogenesis by macrophages, dendritic cells, and circulating myelomonocytic cells. Current Pharmaceutical Design 15 365-379. (doi:10.2174/ 138161209787315783)

Dekel N, Gnainsky Y, Granot I \& Mor G 2010 Inflammation and implantation. American Journal of Reproductive Immunology 63 17-21. (doi:10.1111/ j.1600-0897.2009.00792.x)

Dekel N, Gnainsky Y, Granot I, Racicot K \& Mor G 2014 The role of inflammation for a successful implantation. American Journal of Reproductive Immunology 72 141-147. (doi:10.1111/aji.12266)

Dominguez F, Martinez S, Quinonero A, Loro F, Horcajadas JA, Pellicer A \& Simon C 2008 CXCL10 and IL-6 induce chemotaxis in human trophoblast cell lines. Molecular Human Reproduction 14 423-430. (doi:10.1093/molehr/gan032)

Edwards RG 1994 Implantation, interception and contraception. Human Reproduction 9 985-995.

El-Toukhy T, Sunkara S \& Khalaf Y 2012 Local endometrial injury and IVF outcome: a systematic review and meta-analysis. Reproductive Biomedicine Online 25 345-354. (doi:10.1016/j.rbmo.2012.06.012)

Fatemi HM \& Popovic-Todorovic B 2013 Implantation in assisted reproduction: a look at endometrial receptivity. Reproductive Biomedicine Online 27 530-538. (doi:10.1016/j.rbmo.2013.05.018)

Geissmann F, Manz MG, Jung S, Sieweke MH, Merad M \& Ley K 2010 Development of monocytes, macrophages, and dendritic cells. Science 327 656-661. (doi:10.1126/science.1178331)

Genbacev OD, Prakobphol A, Foulk RA, Krtolica AR, Ilic D, Singer MS, Yang ZQ, Kiessling LL, Rosen SD \& Fisher SJ 2003 Trophoblast L-selectin-mediated adhesion at the maternal-fetal interface. Science 229 405-408. (doi:10.1126/science.1079546)

Gibreel A, Badawy A, El-Refai W \& El-Adawi N 2013 Endometrial scratching to improve pregnancy rate in couples with unexplained subfertility: a randomized controlled trial. Journal of Obstetrics and Gynaecology Research 39 680-684. (doi:10.1111/j.1447-0756.2012. 02016.x)

Gipson IK, Blalock T, Tisdale A, Spurr-Michaud S, Allcorn S, StavreusEvers A \& Gemzell K 2008 MUC16 is lost from the uterodome (pinopode) surface of the receptive human endometrium: in vitro evidence that MUC16 is a barrier to trophoblast adherence. Biology of Reproduction 78 134-142. (doi:10.1095/biolreprod.106.058347)

Gnainsky Y, Granot I, Aldo PB, Barash A, Or Y, Schechtman E, Mor G \& Dekel N 2010 Local injury of the endometrium induces an inflammatory response that promotes successful implantation. Fertility and Sterility $\mathbf{9 4}$ 2030-2036. (doi:10.1016/j.fertnstert.2010.02.022)
Goetzl EJ, Banda MJ \& Leppert D 1996 Matrix metalloproteinases in immunity. Journal of Immunology 156 1-4.

Granot I, Gnainsky Y \& Dekel N 2012 Endometrial inflammation and effect on implantation improvement and pregnancy outcome. Reproduction 144 661-668. (doi:10.1530/REP-12-0217)

Hanlon DJ, Aldo PB, Devine L, Alvero AB, Engberg AK, Edelson R \& Mor G 2011 Enhanced stimulation of anti-ovarian cancer CD8(+) T cells by dendritic cells loaded with nanoparticle encapsulated tumor antigen. American Journal of Reproductive Immunology 65 597-609. (doi:10. 1111/j.1600-0897.2010.00968.x)

Hannan NJ, Jones RL, White CA \& Salamonsen LA 2006 The chemokines, CX3CL1, CCL14, and CCL4, promote human trophoblast migration at the feto-maternal interface. Biology of Reproduction 74 896-904. (doi:10.1095/biolreprod.105.045518)

Horcajadas JA, Pellicer A \& Simón C 2007 Wide genomic analysis of human endometrial receptivity: new times, new opportunities. Human Reproduction Update 13 77-86. (doi:10.1093/humupd/dml046)

Illera MJ, Cullinan E, Gui Y, Yuan L, Beyler SA \& Lessey BA 2000 Blockade of the $\alpha(v) \beta(3)$ integrin adversely affects implantation in the mouse. Biology of Reproduction 62 1285-1290. (doi:10.1095/biolreprod62.5.1285)

Illera MJ, Lorenzo PL, Gui YT, Beyler SA, Apparao KB \& Lessey BA 2003 A role for $\alpha v \beta 3$ integrin during implantation in the rabbit model. Biology of Reproduction $\mathbf{6 8} 766-771$.

Iwamoto S, Iwai S, Tsujiyama K, Kurahashi C, Takeshita K, Naoe M, Masunaga A, Ogawa Y, Oguchi K \& Miyazaki A 2007 TNF- $\alpha$ drives human $\mathrm{CD} 14+$ monocytes to differentiate into $\mathrm{CD} 70+$ dendritic cells evoking Th1 and Th17 responses. Journal of Immunology 179 1449-1457. (doi:10.4049/jimmunol.179.3.1449)

Johnson GA, Burghardt RC, Bazer FW \& Spencer TE 2003 Osteopontin: roles in implantation and placentation. Biology of Reproduction 69 1458-1471. (doi:10.1095/biolreprod.103.020651)

Kämmerer U 2005 Antigen-presenting cells in the decidua. Chemical Immunology and Allergy 89 96-104.

Kaneko Y, Day ML \& Murphy CR 2011 Integrin $\beta 3$ in rat blastocysts and epithelial cells is essential for implantation in vitro: studies with Ishikawa cells and small interfering RNA transfection. Human Reproduction 26 1665-1674. (doi:10.1093/humrep/der128)

Karimzadeh MA, Ayazi Rozbahani M \& Tabibnejad N 2009 Endometrial local injury improves the pregnancy rate among recurrent implantation failure patients undergoing in vitro fertilisation/intra cytoplasmic sperm injection: a randomised clinical trial. Australian and New Zealand Journal of Obstetrics and Gynaecology 49 677-680. (doi:10.1111/ j.1479-828X.2009.01076.x)

Kitaya K, Nakayama T, Okubo T, Kuroboshi H, Fushiki S \& Honjo H 2003 Expression of macrophage inflammatory protein- $1 \beta$ in human endometrium: its role in endometrial recruitment of natural killer cells. Journal of Clinical Endocrinology and Metabolism 88 1809-1814. (doi:10.1210/jc. 2002-020980)

Krikun G, Mor G, Alvero A, Guller S, Schatz F, Sapi E, Rahman M, Caze R, Qumsiyeh M \& Lockwood CJ 2004 A novel immortalized human endometrial stromal cell line with normal progestational response. Endocrinology 145 2291-2296. (doi:10.1210/en.2003-1606)

Laskarin G, Kämmerer U, Rukavina D, Thomson AW, Fernandez N \& Blois SM 2007 Antigen-presenting cells and materno-fetal tolerance: an emerging role for dendritic cells. American Journal of Reproductive Immunology 58 255-267. (doi:10.1111/j.1600-0897.2007.00511.x)

Lessey BA 2002 Adhesion molecules and implantation. Journal of Reproductive Immunology 55 101-112. (doi:10.1016/S0165-0378(01) 00139-5)

Lessey BA, Damjanovich L, Coutifaris C, Castelbaum A, Albelda SM \& Buck CA 1992 Integrin adhesion molecules in the human endometrium. Correlation with the normal and abnormal menstrual cycle. Journal of Clinical Investigation 90 188-195. (doi:10.1172/JCl115835)

Li R \& Hao G 2009 Local injury to the endometrium: its effect on implantation. Current Opinion in Obstetrics \& Gynecology 21 236-239. (doi:10.1097/GCO.0b013e32832a0654)

Liu N, Zhou C, Chen Y \& Zhao J 2013 The involvement of osteopontin and $\beta 3$ integrin in implantation and endometrial receptivity in an early mouse pregnancy model. European Journal of Obstetrics Gynaecology and Reproductive Biology 170 171-176. (doi:10.1016/j.ejogrb.2013. 06.019) 
Luster AD, Alon R \& von Andrian UH 2005 Immune cell migration in inflammation: present and future therapeutic targets. Nature Immunology 6 1182-1190. (doi:10.1038/ni1275)

Margarit L, Gonzalez D, Lewis PD, Hopkins L, Davies C, Conlan RS, Joels L \& White JO 2009 L-selectin ligands in human endometrium: comparison of fertile and infertile subjects. Human Reproduction 24 2767-2777. (doi:10.1093/humrep/dep247)

McIntire RH, Ganacias KG \& Hunt JS 2008 Programming of human monocytes by the uteroplacental environment. Reproductive Sciences 15 437-447. (doi:10.1177/1933719107314065)

van Mourik MS, Heijnen CJ \& Macklon NS 2009 Embryonic implantation: cytokines, adhesion molecules, and immune cells in establishing an implantation environment. Journal of Leukocyte Biology 85 4-19. (doi:10.1189/jlb.0708395)

Narvekar SA, Gupta N, Shetty N, Kottur A, Srinivas M \& Rao KA 2010 Does local endometrial injury in the nontransfer cycle improve the IVF-ET outcome in the subsequent cycle in patients with previous unsuccessful IVF? A randomized controlled pilot study. Journal of Human Reproductive Sciences 3 15-19. (doi:10.4103/0974-1208.63116)

Nastri CO, Ferriani RA, Raine-Fenning N \& Martins WP 2013 Endometrial scratching performed in the non-transfer cycle and outcome of assisted reproduction: a randomized controlled trial. Ultrasound in Obstetrics \& Gynecology 42 375-382. (doi:10.1002/uog.12638)

Plaks V, Birnberg T, Berkutzki T, Sela S, BenYashar A, Kalchenko V, Mor G Keshet E, Dekel N, Neeman M et al. 2008 Uterine DCs are crucial for decidua formation during embryo implantation in mice. Journal of Clinical Investigation 118 3954-3965. (doi:10.1172/JCI36682)

Potdar N, Gelbaya T \& Nardo LG 2012 Endometrial injury to overcome recurrent embryo implantation failure: a systematic review and meta-analysis. Reproductive Biomedicine Online 25 561-571. (doi:10.1016/j.rbmo.2012.08.005)

Raziel A, Schachter M, Strassburger D, Berno O, Ron-EI R \& Friedler S 2007 Favorable influence of local injury to the endometrium in intracytoplasmic sperm injection patients with high-order implantation failure. Fertility and Sterility 87 198-201. (doi:10.1016/j.fertnstert.2006. 05.062)

Rieger L, Honig A, Sütterlin M, Kapp M, Dietl J, Ruck P \& Kämmerer U 2004 Antigen-presenting cells in human endometrium during the menstrual cycle compared to early pregnancy. Journal of the Society for Gynecologic Investigation 11 488-493. (doi:10.1016/j.jsgi.2004.05.007)

Schober A \& Weber C 2005 Mechanisms of monocyte recruitment in vascular repair after injury. Antioxidants \& Redox Signaling 7 1249-1257. (doi:10.1089/ars.2005.7.1249)
Sela HY, Goldman-Wohl DS, Haimov-Kochman R, Greenfield C, NatansonYaron S, Hamani Y, Revel A, Lavy Y, Singer O, Yachimovich-Cohen N et al. 2013 Human trophectoderm apposition is regulated by interferon $\gamma$-induced protein 10 (IP-10) during early implantation. Placenta 34 222-230. (doi:10.1016/j.placenta.2012.12.008)

Sentman CL, Meadows SK, Wira CR \& Eriksson M 2004 Recruitment of uterine NK cells: induction of CXC chemokine ligands 10 and 11 in human endometrium by estradiol and progesterone. Journal of Immunology 173 6760-6766. (doi:10.4049/jimmunol.173.11.6760)

Seymour JF, Lieschke GJ, Grail D, Quilici C, Hodgson G \& Dunn AR 1997 Mice lacking both granulocyte colony-stimulating factor (CSF) and granulocyte-macrophage CSF have impaired reproductive capacity, perturbed neonatal granulopoiesis, lung disease, amyloidosis, and reduced long-term survival. Blood 90 3037-3049.

Shi C \& Pamer EG 2011 Monocyte recruitment during infection and inflammation. Nature Reviews. Immunology 11 762-774. (doi:10.1038/ nri3070)

Simon C, Moreno C, Remohi J \& Pellicer A 1998 Molecular interactions between embryo and uterus in the adhesion phase of human implantation. Human Reproduction 13 219-232. (doi:10.1093/humrep/13.suppl_3.219)

Tiboni GM, Giampietro F, Gabriele E, Di Donato V \& Impicciatore GG 2011 Impact of a single endometrial injury on assisted reproductive technology outcome: a preliminary observational study. Journal of Reproductive Medicine 56 504-506.

Zhou L, Li R, Wang R, Huang HX \& Zhong K 2008 Local injury to the endometrium in controlled ovarian hyperstimulation cycles improves implantation rates. Fertility and Sterility 89 1166-1176. (doi:10.1016/ j.fertnstert.2007.05.064)

Ziebe S, Loft A, Povlsen BB, Erb K, Agerholm I, Aasted M, Gabrielsen A, Hnida C, Zobel DP, Munding B et al. 2013 A randomized clinical trial to evaluate the effect of granulocyte-macrophage colony-stimulating factor (GM-CSF) in embryo culture medium for in vitro fertilization. Fertility and Sterility 99 1600-1609. (doi:10.1016/j.fertnstert.2012.12.043)

Received 7 August 2014

First decision 10 September 2014

Revised manuscript received 22 October 2014

Accepted 27 October 2014 